\title{
Interrogation of Fabry-Pérot ultrasound sensors with Bessel beams
}

Sheppard, Oliver, Guggenheim, James, Marques, Dylan, Ansari, Rehman, Zhang, Edward, et al.

Oliver J. Sheppard, James A. Guggenheim, Dylan M Marques, Rehman Ansari, Edward Z Zhang, Paul C. Beard, Peter R. T. Munro, "Interrogation of Fabry-Pérot ultrasound sensors with Bessel beams," Proc. SPIE 11240, Photons Plus Ultrasound: Imaging and Sensing 2020, 1124014 (5 March 2020); doi: $10.1117 / 12.2551305$

SPIE. Event: SPIE BiOS, 2020, San Francisco, California, United States 


\title{
Interrogation of Fabry-Pérot ultrasound sensors with Bessel beams
}

\author{
Oliver J. Sheppard ${ }^{\mathrm{a}}$, James A. Guggenheim ${ }^{\mathrm{a}}$, Dylan M. Marques ${ }^{\mathrm{a}}$, Rehman Ansari ${ }^{\mathrm{a}}$, Edward \\ Z. Zhang ${ }^{\mathrm{a}}$, Paul C. Beard ${ }^{\mathrm{a}}$, and Peter R. T. Munro ${ }^{\mathrm{a}}$ \\ aDepartment Medical Physics \& Biomedical Engineering, University College London, UK
}

\begin{abstract}
Fabry-Pérot etalon-based ultrasound detectors are typically interrogated with a focused Gaussian beam in order to achieve a desired acoustic element size. However, tightly focused Gaussian beams lead to beam 'walk-off' within the etalon cavity which reduces sensitivity. In previous work, the planar geometry of the Fabry-Pérot etalon has been replaced by a curved geometry matched to the interrogation beam geometry, thus preventing walk-off. In this work we instead propose using propagation invariant Bessel beams, thus matching the beam geometry to that of the planar etalon geometry, to reduce beam walk-off and increase sensitivity. Increased sensitivity may extend the imaging depth of Fabry-Pérot ultrasound detection systems and may thus enable photoacoustic imaging to access a range of deep tissue imaging applications.
\end{abstract}

Keywords: Bessel Beam, Fabry-Pérot Etalons, Sensors, Sensitivity, Photoacoustic imaging, Beam Confinement

\section{INTRODUCTION}

Photoacoustic (PA) imaging is a biomedical imaging technique which combines the high contrast derived from light-absorption in biological tissue with the high spatial resolution of ultrasound imaging. ${ }^{1}$ In particular, ultrasound pressure waves are generated in tissue, via the PA effect, when light is absorbed by chromophores. Detecting the ultrasound waves with an array of sensors allows for the creation of a 3D map of the initial pressure distribution which may be linked with chromophore concentration. ${ }^{2}$ In order to obtain high spatial resolution 3D maps deep in tissue, it is desirable to have a sensor that: is highly sensitive to ultrasound waves, has a small effective acoustic element size, and has a uniform frequency response. The Fabry-Pérot (FP) etalon-based ultrasound sensor is one such sensor which satisfies these criteria. High fidelity PA images with an imaging depth up to $1 \mathrm{~cm}$ have been produced with this sensor (see for example Jathoul et. $\mathrm{al}^{3}$ ). However, some clinically important biological structures, such as some lymph nodes and breast tumours, lie just beyond this imaging depth. In order to potentially increase this imaging depth, and therefore the clinical usability of the sensors, it is necessary to increase the sensor's sensitivity. In this proceedings we describe an approach to increasing the sensitivity of FP ultrasound sensors using Bessel interrogation beams, instead of Gaussian interrogation beams which have been used up until now.

The proceedings begins with an introduction to the FP sensor, how its sensitivity may be defined and the factors which effect it. We then introduce Bessel beams as a potential interrogation beam for FP sensors. We explain how the optical readout of FP sensors with Bessel beams can be modelled, before providing examples and experimental validation. The proceedings concludes with a comparison of sensitivity for Bessel and Gaussian interrogation beams.

Further author information:

O.J.S.: E-mail: oliver.sheppard.15@ucl.ac.uk

Photons Plus Ultrasound: Imaging and Sensing 2020, edited by Alexander A. Oraevsky, Lihong V. Wang, Proc. of SPIE Vol. 11240, 1124014 · @ 2020 SPIE - CCC code: 1605-7422/20/\$21 · doi: 10.1117/12.2551305 


\subsection{Planar Fabry-Pérot ultrasound sensors}

FP etalons are optical resonators formed by two parallel mirrors surrounding a cavity. When used as an ultrasound sensor the time dependent intensity of a reflected or transmitted beam is detected. A reflection geometry is considered in this work and so we neglect the transmission case from this point on. Up until now, interrogation beams have generally had a Gaussian transverse profile. By choosing an appropriate wavelength for the interrogation beam, the intensity of the reflected interrogation beam can be made to vary in response to ultrasound waves which modulate the thickness of the cavity.

This modulation of an etalon's reflectivity in response to an incident ultrasound wave is usually characterized by the interferometer transfer function (ITF), which is the reflected interrogation beam intensity measured as a function of wavelength. Example ITFs are shown in Fig. 1. A resonance condition occurs when the double pass optical path length of the cavity is equal to an integer number of wavelengths. This resonance leads to the reflectivity of the etalon being highly sensitive to changes to the cavity thickness. When an ultrasound wave perturbs the cavity thickness, and therefore the optical path length within the cavity, the cavity resonance wavelength must also change. The wavelength of the interrogation beam, known as the bias wavelength, is chosen to maximise the sensitivity. At the bias wavelength, the change in resonance wavelength can be seen as a change in the reflected intensity, which can be related to the pressure of the ultrasound wave.
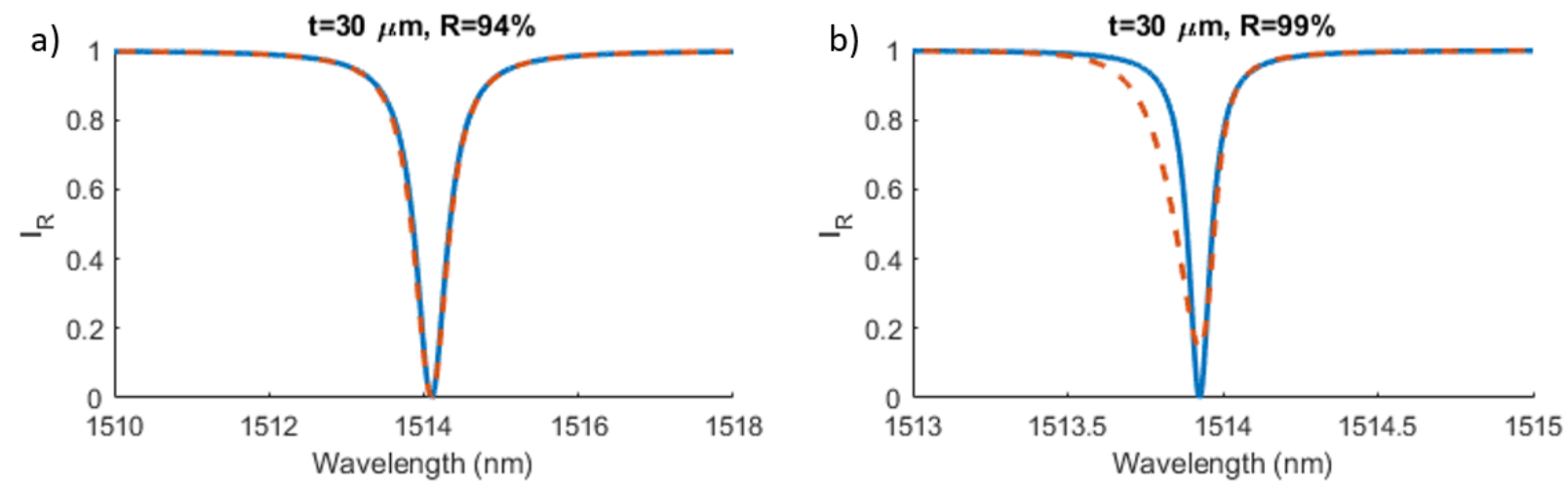

$$
\begin{aligned}
& \text { Collimated Gaussian }(2 \mathrm{w}=250 \mu \mathrm{m}) \\
& -\quad-\text { Focussed Gaussian }(2 \mathrm{w}=50 \mu \mathrm{m})
\end{aligned}
$$

Figure 1. a) A typical ITF from a sensor with a cavity length of $30 \mu \mathrm{m}$ and mirror reflectivity of $94 \%$ for both a collimated and a focused Gaussian beam. b) The same ITF if the mirror reflectivity is increased to $99 \%$

The sensitivity of this sensor is a combination of the acoustic and optical sensitivities. The acoustic sensitivity is determined by how strongly ultrasound waves modulate the cavity thickness, whilst the optical sensitivity is determined by how the optical reflectivity of the etalon changes in response to a change in cavity thickness. One metric for describing optical sensitivity is: $\left|\frac{d I_{r}}{d \lambda}\right|$, where $I_{r}$ is the reflected interrogation beam intensity ${ }^{2}$ and the bias wavelength is chosen to maximise this metric. However, due to noise contributions such as dark current and shot noise, this metric is not always appropriate. In general, it is desirable for an ITF to have a steep transition between the lowest and highest reflected intensity, whilst maintaining a significant difference between these two intensities. To reflect these requirements the following metric has been shown to be effective for quantifying optical sensitivity:

$$
\left|\frac{d I_{r}}{d \lambda} / \sqrt{I_{r}}\right|
$$

In addition to optical sensitivity, some other figures of merit are also commonly used to describe and compare ITFs. One such metric is visibility, which is defined as $V=\left(I_{\max }-I_{\min }\right) /\left(I_{\max }+I_{\min }\right)$, where $I_{\max }$ and $I_{\min }$ are the maximum and minimum reflected intensities, respectively. Another such metric is the full width half maximum (FWHM) of the fringe.

When an etalon is illuminated by a plane wave, the ITF is accurately described by the Airy function, ${ }^{4}$ which predicts that one way to increase sensitivity is to increase the mirror reflectivities. However, a focused beam 
is required to achieve a small acoustic element size if the etalon is to be used as an ultrasound sensor. This requirement limits the ITF slope and visibility due to beam walk-off, an effect where the beam diverges inside the cavity whilst undergoing multiple reflections. ${ }^{5}$ This effect is further exacerbated for longer cavities, higher mirror reflectivities (as seen in Fig. 1 b) ), or more highly divergent beams, such as those with smaller focal spots.

Previous attempts to limit beam walk-off have, to date, focused on changing the cavity shape, either by introducing waveguides into the cavity ${ }^{6}$ or by changing the cavity shape to be planoconcave. ${ }^{7}$ However, such approaches increase the complexity of the manufacturing process, and limits the positions at which the sensor can be interrogated. To improve the lateral beam confinement in a planar FP sensor, another approach is to use an interrogation beam that maintains a small focal spot size over a longer propagation distance. Bessel beams possess an extended focal depth, relative to Gaussian beams, and are thus well suited for use as an interrogation beam. Bessel beams have been used in a range of imaging modalities including optical coherence tomography ${ }^{8-11}$ and light sheet microscopy ${ }^{12,13}$ where their extended depth of focus have been advantageous.

\subsection{Bessel Beams}

Bessel beams, so called because of their transverse field distributions are described by Bessel functions, ${ }^{14}$ possess a central core surrounded by concentric rings, in addition to an extended depth of focus. In this proceedings we compare the transverse spot size of both Gaussian and Bessel beams using FWHM. ${ }^{15}$ Figure 2 shows a comparison between a Gaussian Beam and a Bessel beam with the same FWHM and numerical aperture emphasising the extended focal depth of Bessel beams.

a)
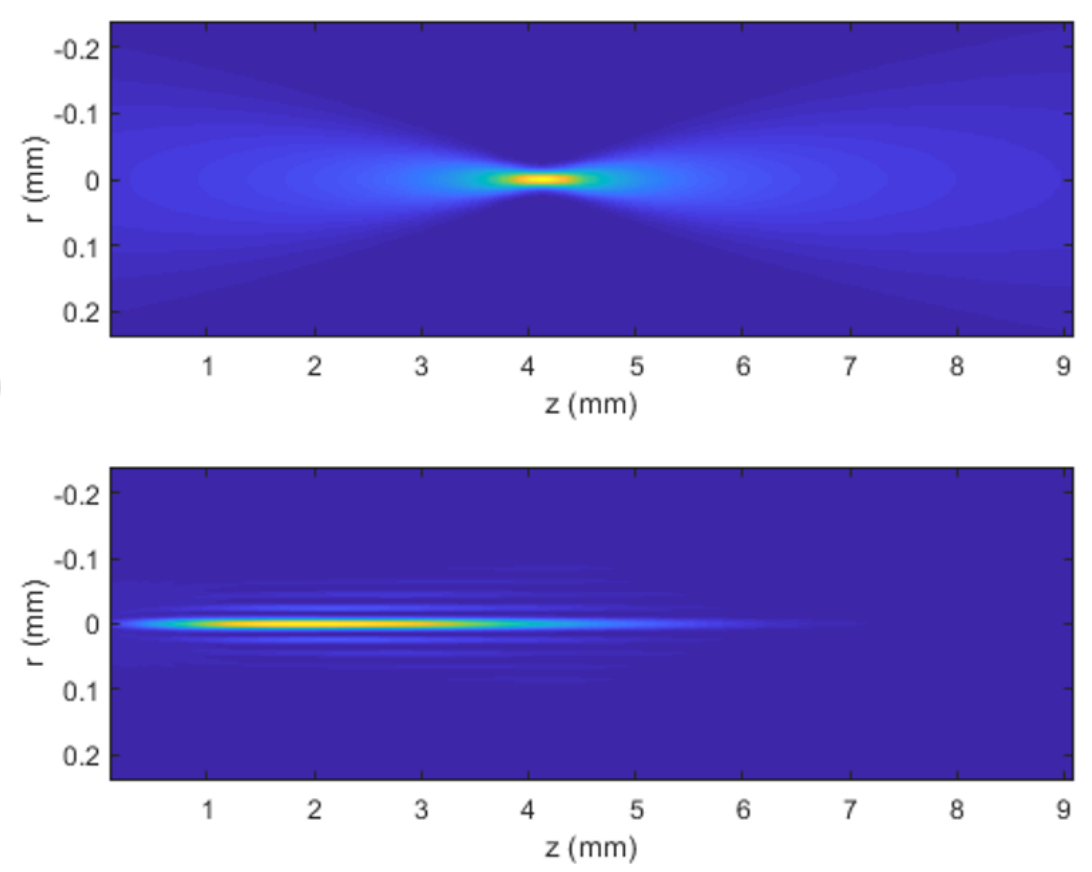

Figure 2. A comparison of Gaussian (a) and Bessel beams (b) with the same FWHM and NA in the (r,z) plane

Different methods have been used to generate Bessel beams including spatial light modulators, ring apertures focused placed in the Fourier plan of a focusing lens, and conical lenses called axicons, as were used in this work. The field distribution produced by an axicon can be described by: ${ }^{16}$

$$
E(r, z)=-i \frac{k}{z} \exp \left[i \frac{k}{2 z} r^{2}\right] \int_{0}^{\infty} t\left(r^{\prime}\right) \exp \left[i \frac{k}{2 z} r^{\prime 2}\right] J_{0}\left[\frac{k r}{z} r^{\prime}\right] d r^{\prime}
$$


where $k$ is the optical wavenumber $(k=2 \pi / \lambda),(r, z)$ are the radial and longitudinal axial coordinates in the focal region, $r^{\prime}$ is the radial coordinate at the entrance face of the axicon, $J_{0}$ is the zeroth order Bessel function of the first kind, and $t\left(r^{\prime}\right)$ is the transmittance function of the axicon, i.e., the field that illuminates the back face of the axicon multiplied by the phase structure applied by the axicon. For example, the transmittance function of an axicon with a numerical aperture (NA) of $\beta$, illuminated by a Gaussian beam of $1 / e^{2}$ radius $w$, is given by: ${ }^{8}$

$$
t\left(r^{\prime}\right)=\sqrt{\frac{2}{\pi}} \frac{1}{w} \exp \left[-\left(\frac{r^{\prime}}{w}\right)^{2}\right] \exp \left[-i k \beta r^{\prime}\right]
$$

We note that $\beta$ is related to the wedge angle of the axicon, $\alpha$, and the refractive index of the axicon, $n$, according to $\beta=(n-1) \alpha$. A ray diagram illustrating this example can be seen in Fig. 3. For such a field the spot size is related to the axicon's NA and the incident wavelength only, ${ }^{14}$ because the radial position of the field's first zero is given by:

$$
r_{0}=\frac{2.405}{k_{r}}=\frac{2.405}{k \sin \beta}
$$

and this is what we will use to define the Bessel beam spot size. Furthermore, the length of the focal zone is related to the axicon's $\beta$ and $w$ values as: ${ }^{8}$

$$
L_{w}=w / \beta .
$$

When a Bessel beam is magnified by a 4 f system with a magnification $M$, where the magnified spot size becomes $M r_{0}$, the effective focal depth becomes $M^{2} L_{w}$. These relationships mean that, unlike Gaussian beams, the spot size and the focal depth for Bessel beams are somewhat decoupled. The implication of this is beam walk-off can be reduced whilst increasing etalon optical sensitivity.

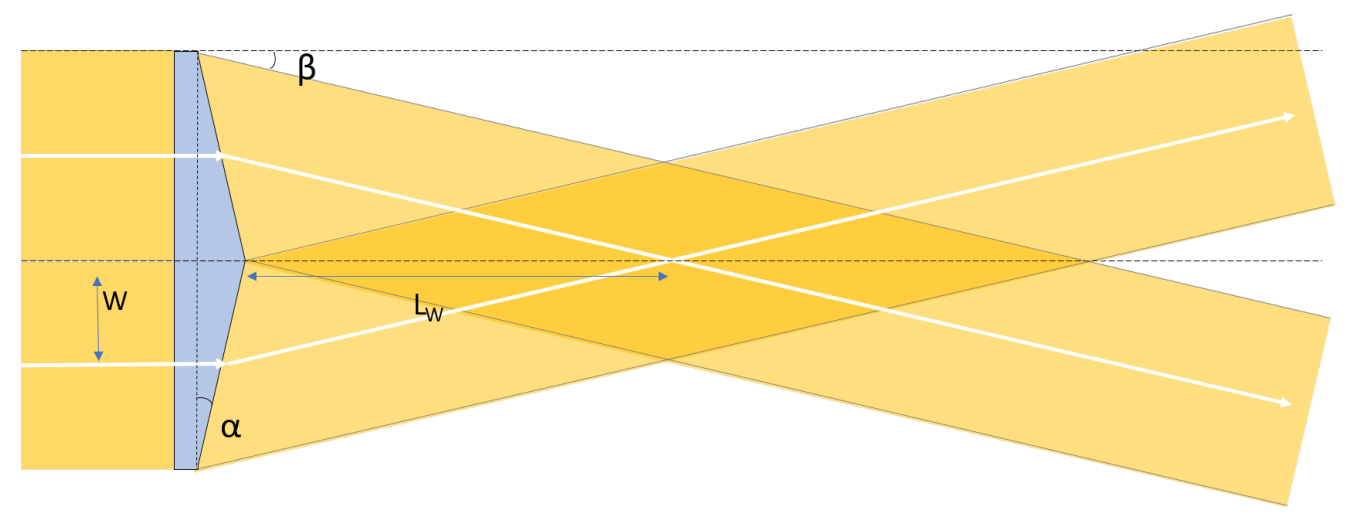

Figure 3. Ray diagram of an axicon with wedge angle of $\alpha$ and an NA of $\beta$, illuminated by a Gaussian beam with radius $w$ producing a Bessel beam with focal depth of $L_{w}$.

\section{MODEL}

A schematic diagram of the system modelled in this paper is shown in Fig. 4. A tunable wavelength laser source is coupled into an optical fibre. The beam exiting the fibre is collimated before being focused by an axicon to produce a Bessel beam as described by Eqs. 2 and 3. The Bessel beam is reflected by the etalon before propagating back through the axicon and collimator. The reflected beam is then coupled back into the fibre towards the photodetector which measures the power of the reflected beam. The reflected power is recorded for a range of wavelengths allowing an ITF to be acquired.

The system is modelled using by adapting the model reported by Marques et al. ${ }^{5}$ The model is applicable to arbitrary incident beams and works by decomposing such beams into plane waves, each propagating at different angles with an associated complex amplitude. ${ }^{17}$ The distribution of plane waves is known as an angular spectrum. The propagation angle denotes the angle that the plane wave makes relative to the FP etalon. In our model we 
consider the angular spectrum of the Bessel beam generated when a Gaussian beam with $1 / e^{2}$ radius is incident upon an axicon with refractive index $n$ and a wedge angle of $\alpha$.

The angular spectrum is used because the interaction between a single each plane wave and the FP etalon may be calculated without approximation using the theory of optical interference in thin films. ${ }^{18}$ This allows for the amplitude and phase of the reflected wave to be calculated for each plane wave incident upon the etalon, thus allowing for the reflected beam to be synthesised from the reflected angular spectrum. The reflected beam is then propagated back through the axicon and the amount of light coupled back into the fibre calculated. An ITF is calculated by repeating this over a range of wavelengths.

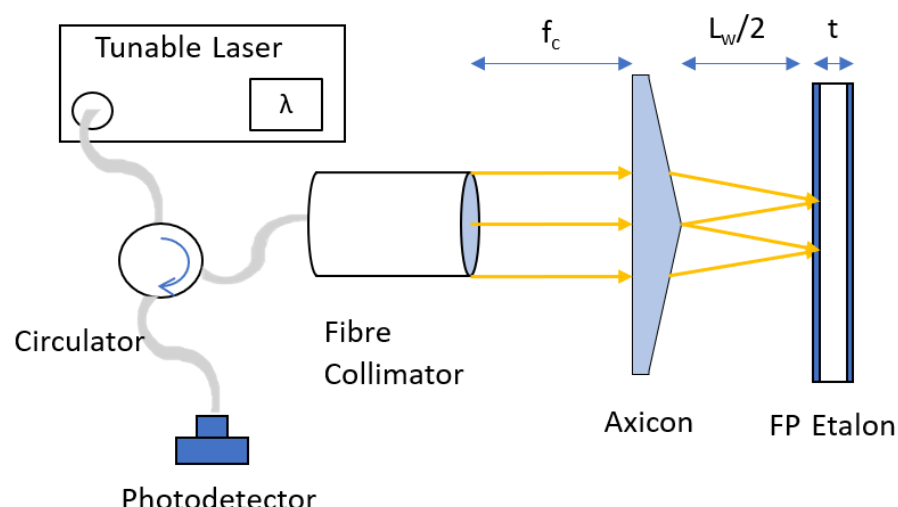

Figure 4. A schematic of the modelled and experimental optical setup.

\subsection{Experimental validation}

The experimental setup described in Sec. 2 and illustrated in Fig. 4 was used to validate the model. A wavelength tunable laser (Santec TSL-510) with a tuning range between $1510 \mathrm{~nm}$ and $1620 \mathrm{~nm}$ was used. Within this wavelength range the mirrors of the custom made etalon had reflectivities in the range of $98 \%$ to $99.55 \%$ allowing us to perform experiments for multiple mirror reflectivities with a single FP etalon. The etalon spacer was made of fused silica and was $102 \mu \mathrm{m}$ thick. $^{5}$ Two different axicons were used to validate the model: one with a wedge angle of $1^{\circ}$ producing a $2 r_{0}$ calcualted from Eq. 4 spot size of $160 \mu \mathrm{m}$, and another with a wedge angle of $5^{\circ}$ producing a $2 r_{0}$ spot size of $32 \mu \mathrm{m}$. Both axicons were illuminated with a $1.5 \mathrm{~mm} 1 / e^{2}$ radius collimated Gaussian beam. The fringes produced by this system, both experimentally and modelled (a sample of which are shown in Fig. 5 and Fig. 6), were characterised by their visibility and FWHM. This was done for both axicons. Different mirror reflectivities were obtained by varying the wavelength within that provided by the tunable laser. The results of these FWHM and visibility simulations and experiments are presented in Figs. 7 and 8. These figures also show a comparison with a Gaussian beam with the same FWHM as the Bessel beams, to give an indication of the improvement these Bessel beams provide. A model developed by Marques et al., ${ }^{5}$ which is well validated for this etalon, was used the calculate the Gaussian beam results.

The results in Figs. 5 to 8 provide preliminary validation of the model. They also suggest that, for the etalon used, Bessel interrogation beams lead to a superior ITF, with higher visibility, and smaller FWHM in all cases, even at the higher mirror reflectivities. The visibility of the fringes produced by Bessel interrogation beams of both spot sizes, vary only weakly with mirror reflectivity, remaining close to a desired visibility of 1 , whilst the visibility of the fringes produced by a Gaussian interrogation beam decrease more rapidly as mirror reflectivity increases. It can also be seen that the visibility and FWHM for Bessel beams are similar for both spot sizes. In contrast, when using Gaussian beams we see a drop by about a factor of 4 in visibility and an increase by about a factor of 5 as the mirror reflectivity increases across the total range considered. These results show that, as expected, decoupling spot size and focal depth, as is possible with Bessel beams, allows for improved ITFs at smaller spot sizes. 

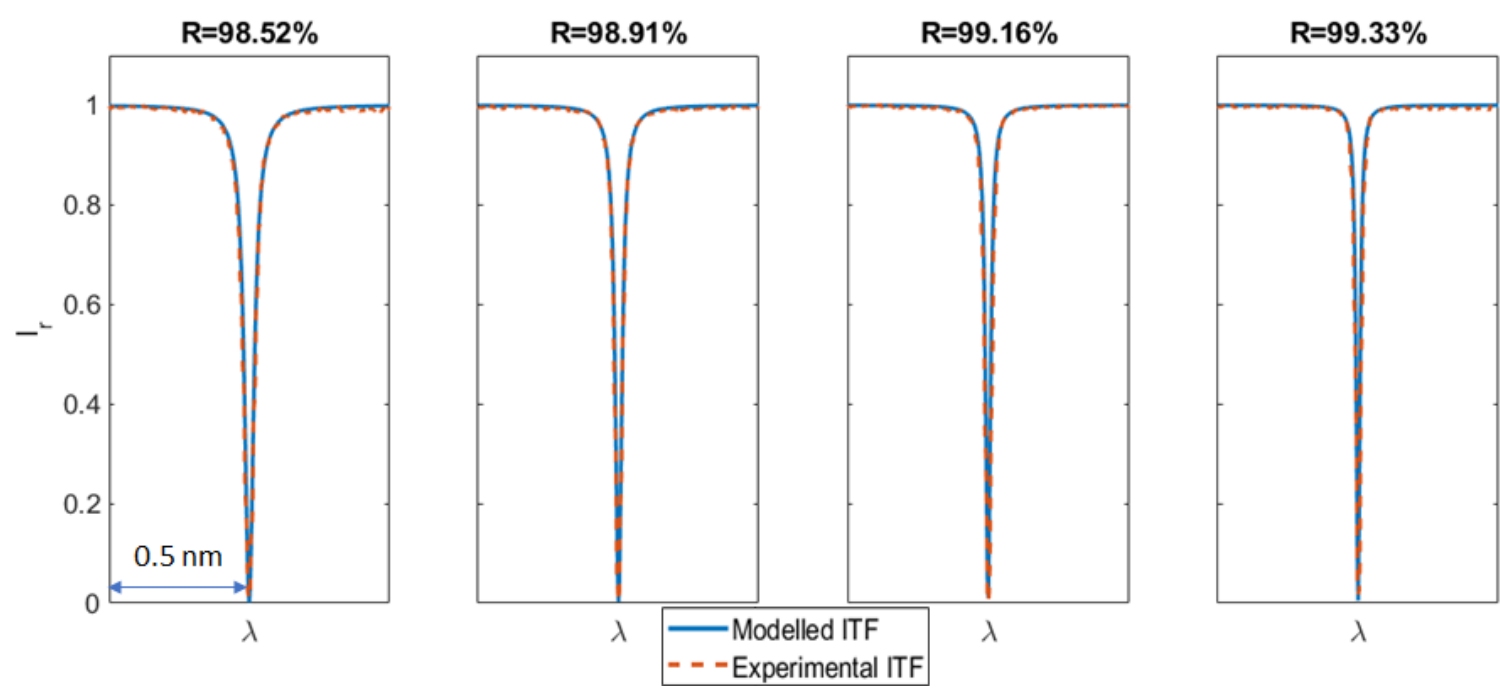

Figure 5. Examples of the modelled and experimental fringes at increasing mirror reflectivities produced by the $1^{\circ}$ axicon giving a $160 \mu \mathrm{m} 2 r_{0}$ spot. The scale bar is equivalent for each fringe.
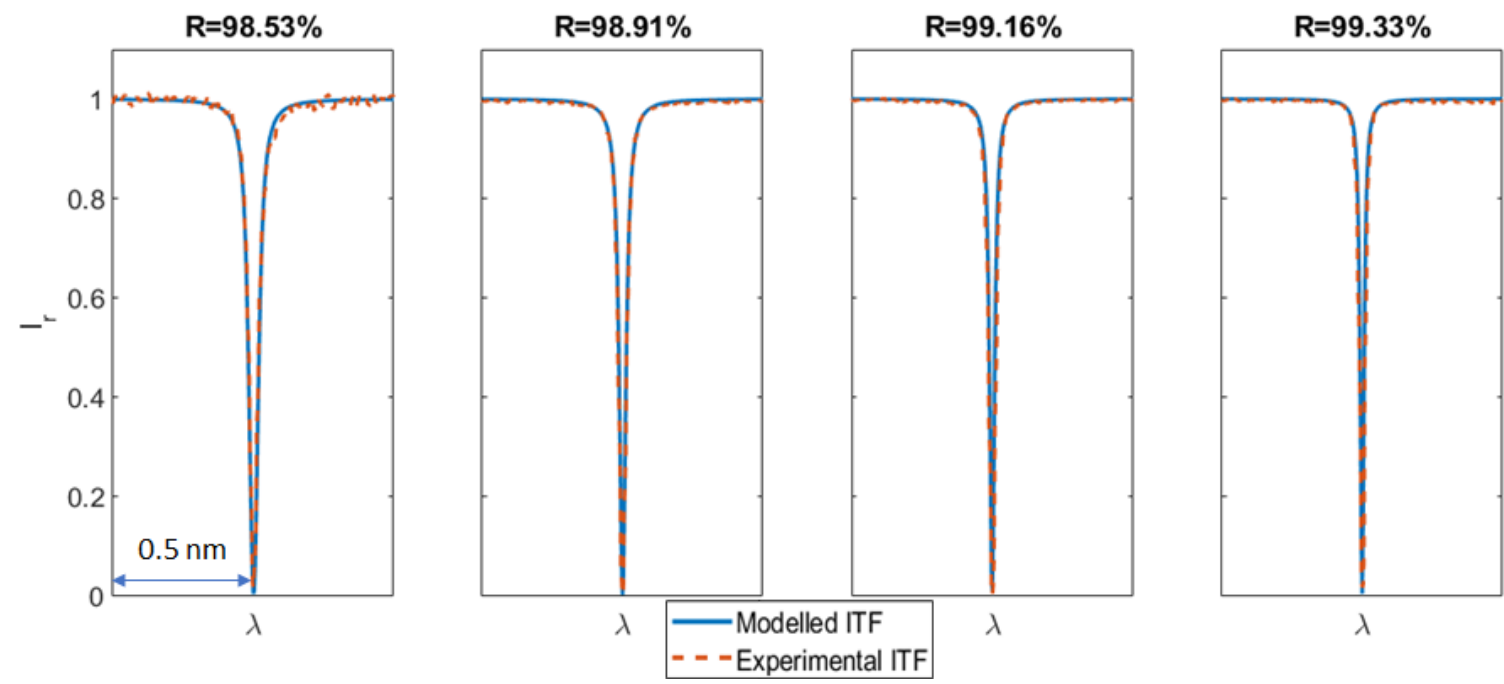

Figure 6. Examples of the modelled and experimental fringes at increasing mirror reflectivities produced by the $5^{\circ}$ axicon giving a $32 \mu \mathrm{m} 2 r_{0}$ spot. The scale bar is equivalent for each fringe.

\section{EFFECT OF BESSEL BEAMS ON SENSITIVITY}

If we consider the $32 \mu \mathrm{m} 2 r_{0}$ Bessel beam and the etalon from the model validation section (Subsec. 2.1), and model magnification via a $4 \mathrm{f}$ system to change the spot size, we can investigate in more detail how spot size effects the sensitivity. Experience with Gaussian interrogation beams suggests that sensitivity should decrease with decreasing spot size, due to increasing beam walk-off. We also expect the difference in sensitivity between the two mirror reflectivities to decrease with spot size for the same reason. We expect Bessel beams to have higher sensitivities than for the associated Gaussian beam case, particularly at smaller spot sizes. We also expect the sensitivity to degrade much less rapidly, as mirror reflectivity increases, for Bessel beams compared with Gaussian beams.

These anticipated relationship are seen clearly in Fig. 9 with the sensitivity increasing by a factor of only approximately 1.3 at the smallest spot size, for a Gaussian beam, when the reflectivity increases the lowest to highest values considered. This factor of increase is approximately 3 for Bessel beams. At this spot size, the 
a)

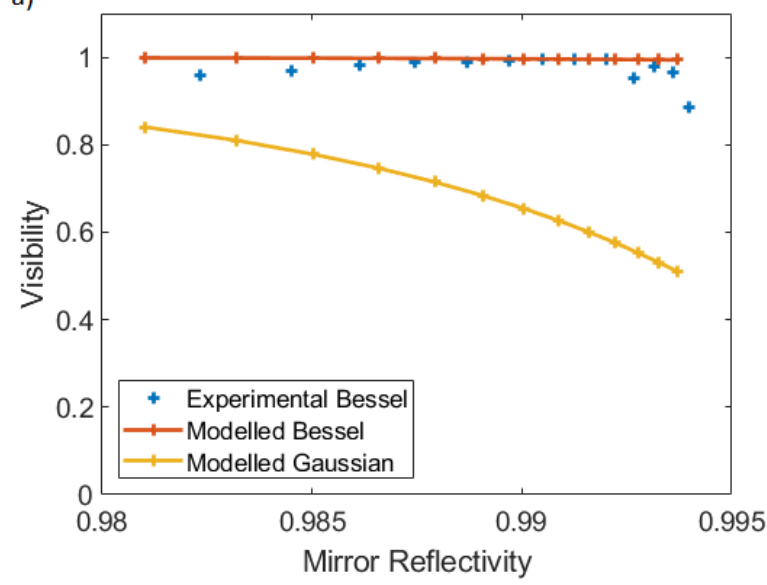

b)

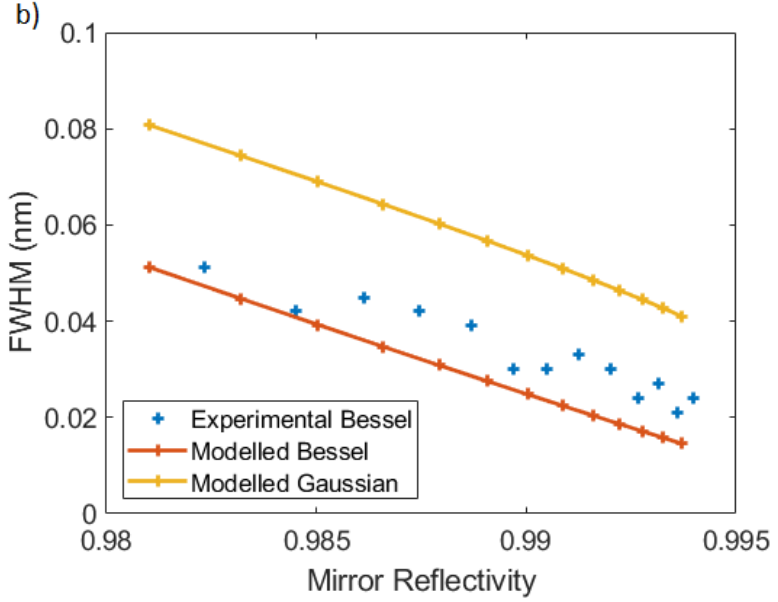

Figure 7. a) The visibility of a fringe at a given mirror reflectivity for a fused silica cavity of $100 \mu \mathrm{m}$ thick produced with a $2 r_{0}$ spot size of $160 \mu \mathrm{m}$. b) the FWHM of a fringe at a given mirror reflectivity for a fused silica cavity produce a $2 r_{0}$ spot size of $160 \mu \mathrm{m}$

a)

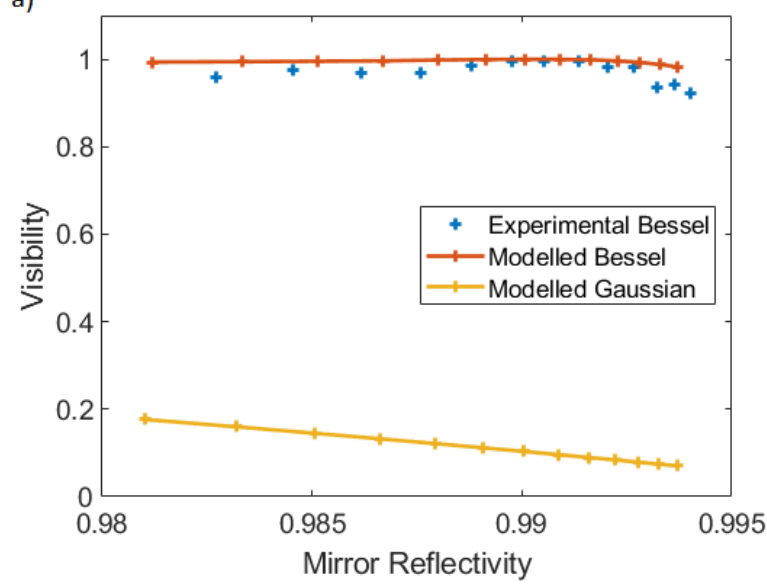

b)

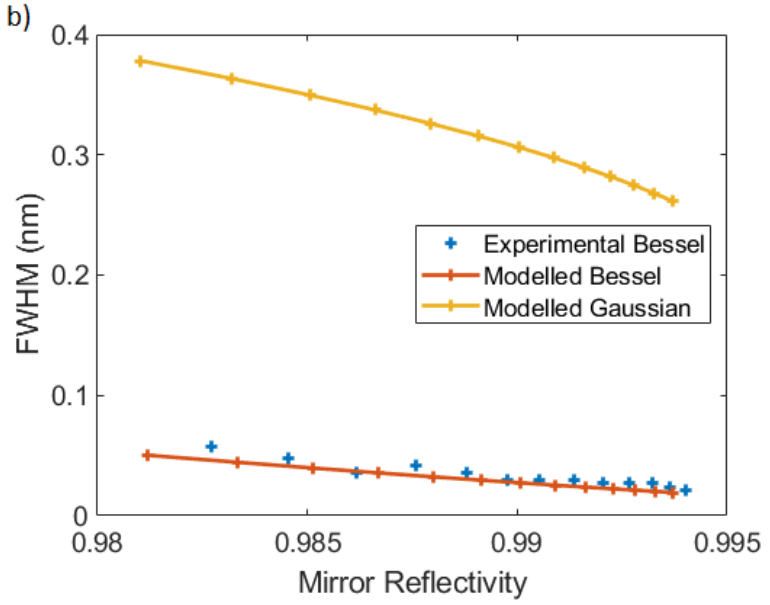

Figure 8. a) The visibility of a fringe at a given mirror reflectivity for a fused silica cavity of $100 \mu \mathrm{m}$ thick produced with a $2 r_{0}$ spot size of $32 \mu \mathrm{m}$. b) the FWHM of a fringe at a given mirror reflectivity for a fused silica cavity produce a $2 r_{0}$ spot size of $32 \mu \mathrm{m}$

maximum sensitivity of the sensor is increased by a factor of 15 by using a Bessel interrogation beam instead of a Gaussian. It should be noted that the Bessel beams and mirror reflectivity could be further optimised for even better sensitivity improvement.

\section{CONCLUSION}

Previously, planar FP ultrasound sensor optical sensitivity was limited by beam walk-off experienced when using focused Gaussian interrogation beams. In this work we have shown that Bessel interrogation beams result in optical sensitivity which is far less dependent on beam spot size, and superior to Gaussian beams for small spot sizes. This study thus demonstrates that by switching from Gaussian interrogation beams to Bessel interrogation beams, optical sensitivity can be maintained even at small spot sizes. We have also shown that, for the fused silica etalon in this study, optical sensitivity may be increased by a factor of 15 simply by using a Bessel, rather than Gaussian, interrogation beam. 


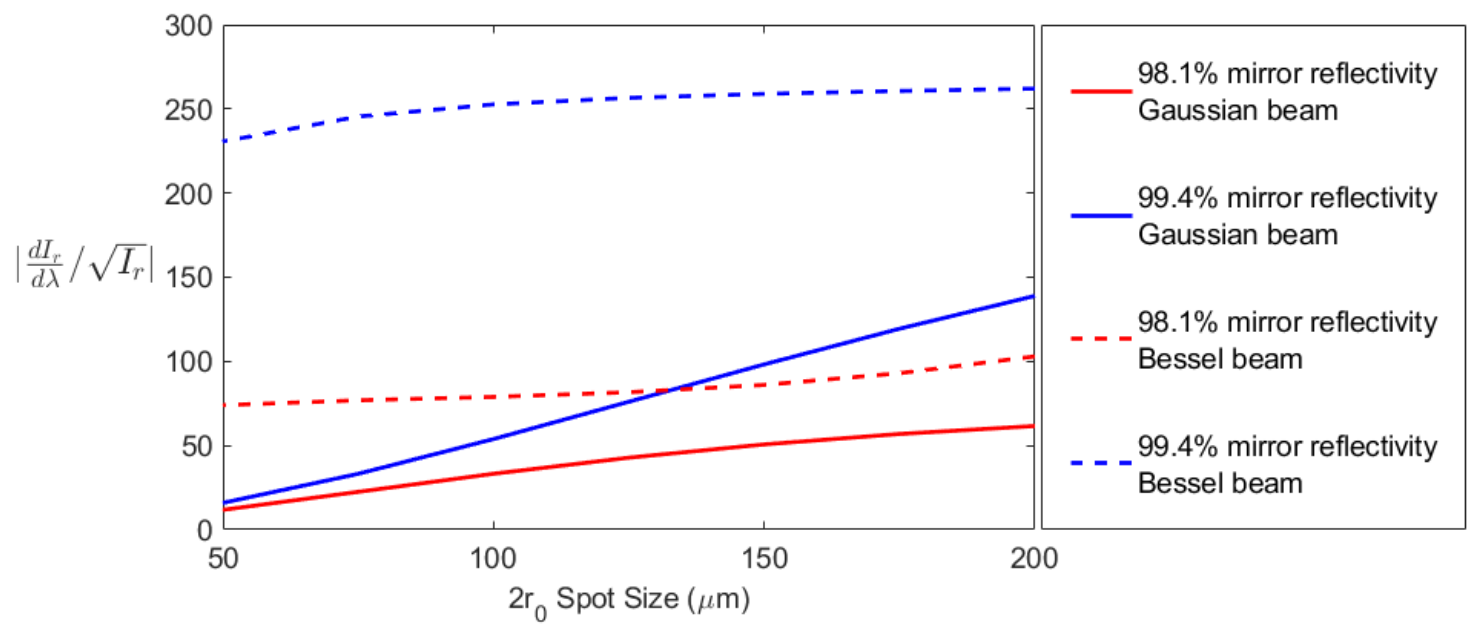

Figure 9. The change in sensitivity parameter $\frac{d I}{d \lambda} / \sqrt{I}$ with $2 r_{0}$ spot size, for Bessel beams and equivalent sized Gaussian beams at two different reflectivities, at different $2 r_{0}$ spot sizes

\section{ACKNOWLEDGMENTS}

The authors acknowledge support from Engineering and Physical Sciences Research Council (EPSRC), European Research Council (ERC) and the joint UCL and Cambridge University Centre for Doctoral Training (CDT) for Integrated Photonic and Electronic Systems (IPES). The authors P. R. T. Munro and J. A. Guggenheim are supported by Royal Society University Research Fellowships. O. J. Sheppard acknowledges the SPIE for their travel grant.

\section{REFERENCES}

[1] Beard, P. C., "Biomedical photoacoustic imaging," Interface Focus 1, 602-631 (2011).

[2] Zhang, E. Z., Laufer, J., and Beard, P., "Backward-mode multiwavelength photoacoustic scanner using a planar fabry-perot polymer film ultrasound sensor for high-resolution three-dimensional imaging of biological tissues," Appl. Opt. 47(4), 561-577 (2008).

[3] Jathoul, A. P., Laufe, J., Ogunlade, O., Treeby, B., Cox, B., Zhang, E., Johnson, P., Pizzey, A. R., Philip, B., Marafioti, T., Lythgoe, M. F., Pedley, R. B., Pule, M. A., and Beard, P., "Deep in vivo photoacoustic imaging of mammalian tissues using a tyrosinase-based genetic reporter," Nat. Photonics 9, 239-246 (2015).

[4] Ismail, N., Kores, C. C., Geskus, D., and Pollnau, M., "Fabry-pérot resonator: spectral line shapes, generic and related airy distributions, linewidths, finesses, and performance at low or frequency-dependent reflectivity," Opt. Express 24(15), 16366-16389 (2016).

[5] Marques, D. M., Guggenheim, J. A., Rehman, A., Zhang, E. Z., Beard, P. C., and Munro, P. R. T., "Modelling fabry-perot illuminated by focussed beams," Opt. Express 28(5), 7691-7706 (2020).

[6] Thathachary, S. V. and Ashkenazi, S., "Toward a highly sensitive polymer waveguide fiber fabry-pérot ultrasound detector," J. Biomed. Opt. 23(10), 106008 (2018).

[7] Guggenheim, J. A., Li, J., Allen, T. J., Colchester, R. J., Noimark, S., Ogunlade, O., Parkin, I. P., Papakonstantinou, I., Desjardins, A. E., Zhang, E. Z., and Beard, P. C., "Ultrasensitive plano-concave optical microresonators for ultrasound sensing," Nat. Photonics 11, 714-719 (2017).

[8] Lorenser, D., Singe, C. C., Curatolo, A., and Sampson, D. D., "Energy-efficient low-fresnel-number bessel beams and their application in optical coherence tomography," Opt. Lett. 39(3), 548-551 (2014).

[9] Ding, Z., Ren, H., Zhao, Y., Nelson, J. S., and Chen, Z., "High-resolution optical coherence tomography over a large depth range with an axicon lens," Opt. Lett. 27(4), 243-245 (2002).

[10] Lee, K.-S. and Rolland, J. P., "Bessel beam spectral-domain high-resolution optical coherence tomography with micro-optic axicon providing extended focusing range," Opt. Lett. 39(15), 1696-1698 (2008). 
[11] Leitgeb, R. A., Villiger, M., Bachmann, A. H., Steinmann, L., and Lasser, T., "Extended focus depth for fourier domain optical coherence microscopy," Opt. Lett. 31(16), 2450-2452 (2006).

[12] Planchon, T. A., Gao, L., Milkie, D. E., Davidson, M. W., Galbraith, J. A., and Betzig, E., "Rapid threedimensional isotropic imaging of living cells using bessel beam plane illumination," Nat. Methods 8, 417-423 (2011).

[13] Fahrbach, F. O., Simon, P., and Rohrbach, A., "Microscopy with self-reconstructing beams," Nat. Photonics 4, 780-785 (2010).

[14] McGloin, D. and Dholakia, K., "Bessel beams: diffraction in a new light," Contemp. Phys. 46(1), 15-28 (2005).

[15] Durnin, J., Miceli, J. J., and Eberly, J. H., "Comparison of bessel and gaussian beams," Opt. Lett. 13(2), 79-80 (1988).

[16] Zapata-Rodríguez, C. J. and Sánchez-Losa, A., "Three-dimensional field distribution in the focal region of low-fresnel-number axicons," J. Opt Soc. Am. A 23(12), 3016-3026 (2006).

[17] Goodman, J. W., [Introduction to Fourier Optics 3rd Ed.], Roberts \& Company, Englewood (2005).

[18] Azzam, R. M. A. and Bashara, N. M., [Ellipsometry and Polarized Light 3rd Ed.], North-Holland, Amsterdam (1999). 